\title{
CÓMO VEN SU FUNCIÓN LOS AUDITORES DE PROCESO AUTOMÁTICO DE DATOS (PAD)
}

\author{
Félix A. Rivera León* \\ E-mail:felixl@hotmail.com
}

\begin{abstract}
RESUMEN
La manera en que los auditores de Proceso Automático de Datos (PAD) ven su función es muy importante para el éxito de cualquier auditoría, ya sea interna, externa o de terceros. Desafortunadamente el administrador de Proceso de Datos (PD) a menudo considera al auditor como "el hombre que dispara a los heridos después de la batalla", mientras que el auditor de PAD percibe su función como de mantenimiento preventivo en el análisis y evaluación de la confiabilidad e integridad de los sistemas de procesamiento de datos.
\end{abstract}

Este artículo discute la manera en que el auditor de PAD y la administración de PD ven la función de auditoria y sugiere cómo pueden trabajar juntos para mejorar esa función.

Palabras clave: Incompatibilidad, fiabilidad, empatía, tabú.

\begin{abstract}
The way in which the PAD auditors see their function is very important for the success of any auditing internal, external or from third people. Unfortunately, the PDA administrador (Data Process) often considers the auditor like the man who shoots the wounded after the battle, while the PAD auditor perceive his function like preventive maintenance in the analysis and evaluation of reliability and integrity of data process systems.

This article discusses the way in which the PAD auditor and the PD administration sees the function of the auditing and suggests how they can work together to improve that function.
\end{abstract}

Key words: Incompatibility, reliability, taboo

* Contador Público Colegiado. Estudios de maestría en Gestión Empresarial.Consultor Nacional e Internacional. Profesor Asociado de la Facultad de Ciencias Administrativas de la Universidad Nacional Mayor de San Marcos. 


\section{INTRODUCCIÓN}

La función de auditoría de Proceso Automático de Datos (PAD) ya no es sólo una actividad auxiliar del departamento de auditoría interna. En la mayor parte de las empresas se ha convertido en una función extremadamente notoria y vital porque los servicios que proporcionan los auditores de PAD son esenciales para el éxito de la compañía.

El reconocimiento general de la necesidad de una función de auditoría de PAD es un fenómeno relativamente reciente. Los resultados de una encuesta llevada a cabo por el Instituto de Investigación de Stanford ${ }^{1}$, indican que el $70 \%$ de las empresas con una función de auditoría de PAD la han tenido tan sólo desde 1970.

La mayor parte de los auditores de PAD ven su función de la misma manera que los auditores internos:

“... para asistir a todos los miembros de la administración (que utilizan las instalaciones del computador o que proveen servicios de computadora) en el desempeño eficaz de sus responsabilidades, brindándoles análisis, evaluaciones y recomendaciones en todas las actividades relacionadas con el Procesamiento de Datos"2.

Además, los auditores de PAD deben poseer las mismas cualidades que sus contrapartes de orientación financiera:

- Habilidad para evaluar objetivamente. Esto entraña un conocimiento funcional de los estándares aceptados en la industria de Proceso de Datos (PD) y la habilidad para comparar operaciones, funciones y procedimientos existentes con aquellos estándares.

- Aptitud para reconocer rápidamente problemas claves. Una función importante de la auditoría es la de identificar fuerzas mayores, problemas fundamentales y deficiencias claves rápidamente (la tarea de distinguir los síntomas de las causas exige habilidades especiales de diagnóstico).

- Capacidad para comunicarse eficazmente. Una cantidad considerable de información debe obtenerse, en un período de tiempo relativamente breve, de administradores, programadores y analistas. Asegurar y comprender esta información sin causar recelo exige fuertes habilidades interpersonales. Además, se necesitan capacidades especiales de comunicación y en el arte de escribir para presentar los hallazgos y las recomendaciones de la auditoría eficazmente.

Sin embargo, de todas las cualidades de la auditoría, la que pone al auditor de PAD aparte del auditor interno es el conocimiento de la función de PD. Puesto que la función de PD envuelve no sólo al departamento de PD sino también a su relación con la administración, los usuarios y los recursos exteriores, es indispensable un conocimiento general del ambiente de PD. Aunque la función de auditoría de PAD es básicamente gerencial más que técnica, los auditores de PAD deben ser bien versados en los conceptos de diseño, operación $\mathrm{y}$ mantenimiento de sistemas.

Aunque las funciones de auditoría de PAD dependen en gran parte de la naturaleza y el tamaño de la instalación de PD (v. gr., proceso centralizado contra unidades en proceso distribuido), los auditores de PAD deberían adoptar un enfoque de auditoría que sea apropiado para los ambientes físico y técnico de la empresa.

\section{RELACIONES DEL AUDITOR DE PAD}

Antes de que los auditores de PAD puedan dirigir programas eficaces de auditoría de PD, deben saber lo que sus empresas esperan de ellos (en particular, la dirección ejecutiva y los departamentos de auditoría interna y de procesamiento de datos). Mientras que los auditores de PD deben comprender claramente los objetivos, las prioridades y necesidades del organismo, la empresa-por su parte- debe entender y apoyar la función de auditoría. La cooperación estrecha entre el auditor de PAD, la dirección ejecutiva, la administración de procesamiento de datos y el auditor interno es esencial para dirigir un programa de auditoría que beneficie a toda la empresa.

\section{Dirección Ejecutiva}

El auditor de PAD y la dirección ejecutiva reciben su autoridad de la Junta Directiva de la empresa: la dirección para diseñar y mantener sistemas de control y para evaluarlos. Puesto que el proceso de evaluación sirve para la función de diseño y mantenimiento, el auditor a menudo es responsable ante la dirección ejecutiva.

El primer paso en el establecimiento de una función de auditoría de PAD es obtener un mandato de la administración superior. A menos que 
los auditores de PAD reciban el apoyo enérgico de la administración superior, puede que no sean capaces de diseñar un programa eficaz de auditoría. El auditor debe, por tanto, convencer a la administración de la necesidad de tal función porque la mayor parte de los administradores están tan involucrados en el logro de las metas y objetivos básicos de la empresa que a menudo descuidan asuntos tales como la seguridad de $\mathrm{PD}^{3}$. El hecho de que la mayor parte de las instalaciones de PD clamen por vigilancia de auditoría no basta para justificar la función de auditoría. El auditor de PAD debe demostrar su capacidad para evaluar situaciones en las que los resultados a menudo son difíciles de medir.

Puesto que la función de auditoría de PAD exige el envolvimiento a nivel ejecutivo con la administración de PD, así como la participación del usuario, ella puede abrir nuevas avenidas de comunicación. El énfasis en los problemas de revisión y de examen concienzudo no sólo contribuyen a resolver esos problemas sino que también demuestran a la administración la capacidad del auditor para discernir las deficiencias de PD.

\section{Auditoria Interna}

El auditor interno y el auditor de PAD llevan a cabo funciones importantes que a menudo son idénticas y se traslapan: el avalúo y el análisis de riesgos así como el análisis de costo/beneficio:

Los auditores internos evalúan la adecuación, la eficiencia y la eficacia de los sistemas de control de una empresa que promueven la eficiencia operativa y fomentan la conformidad con las políticas y otras restricciones establecidas en la compañía. Aunque los auditores internos verifican los resultados informados de la actividad del negocio, principalmente se preocupan de la eficacia continua de los sistemas de control que influyen sobre toda actividad del negocio.

Los auditores de PAD, por otra parte, se preocupan más por:

- La eficacia del departamento de PD.

- La protección del equipo y de los datos contra el uso o el acceso no autorizado.

- La eficiencia de los sistemas existentes.

- El valor económico y la factibilidad de los sistemas en desarrollo.

- La administración de estos recursos.
En la mayor parte de las empresas, la función del auditor de PAD está diseñada para complementar la del auditor interno proporcionando la garantía razonable de que los activos están resguardados, la información es oportuna y confiable, y todos los errores y las deficiencias se descubren y corrigen con prontitud. Mejor control, pistas de auditoría más completas y la conformidad estricta con las políticas de la empresa son igualmente importantes para el auditor de PAD y el auditor interno.

Si el auditor de PAD es un asociado anterior del auditor interno, probablemente será capaz de mantener una buena relación de trabajo con él. Si el auditor de PAD subió a través de las jerarquías del procesamiento de datos y/o es completamente nuevo en el ambiente de auditoría, es probable que se encuentre con alguna forma de "discriminación en sentido contrario". Además, este auditor puede ser vigilado estrictamente para garantizar que en realidad ha cortado sus nexos con el procesamiento de datos. La falta de un título en contabilidad o administración o de experiencia previa en auditoría con una empresa contable también puede impedir la aceptación del auditor de PAD por parte del auditor interno.

El entrenamiento técnico y la idoneidad en PD no siempre garantizan que un auditor de PAD pueda evaluar eficazmente un ambiente de PD; sin embargo, la familiaridad con las prácticas y las normas de auditoría aceptadas, los papeles de trabajo y cosas semejantes pueden ayudar a establecer una buena relación de trabajo con el auditor interno y facilitar una evaluación eficaz de PD.

\section{El administrador de PD}

Mientras que muchos administradores de PD aceptan de mala gana la presencia del auditor en la sala de cómputo, la mayor parte resiente el hecho de que un intruso con poca o ninguna experiencia práctica en PD haya sido asignado para revisar, evaluar y criticar sus operaciones. En cierta manera está justificada su creencia de que pocos auditores son suficientemente conocedores para discutir problemas de PD inteligentemente.

Sin embargo, completar un curso de corta duración sobre conceptos de PD, introductorio de programación, o de diseño y análisis de sistemas, no prepara a los auditores para las condiciones inaceptables que probablemente encontrarán en la sala de cómputo (v. gr., una política de puertas abiertas para programadores y analistas, vaciados 
de memoria en basureros, pruebas con datos reales y así sucesivamente). Mientras que muchas de estas condiciones exigen recomendaciones basadas en la observación personal, el auditor debe evitar proponer soluciones simples que traten de los síntomas en vez de los problemas básicos.

En las empresas en las cuales el auditor de PAD es un ex profesional de PD, la administración de PD puede esperar cierto grado de lealtad y compenetración. Sin embargo, los auditores de PAD deben demostrar su independencia dirigiendo revisiones objetivas de las operaciones y de las aplicaciones de PD, pese a cualquier animosidad que puedan encontrar a consecuencia de ello. Los auditores de PAD pueden contrarrestar estos y otros problemas potenciales de aceptación:

- Explicando al principio el alcance y la función de auditoría.

- Usando estándares reconocidos de la industria de PD como guía.

- Pidiendo la ayuda del personal de PD al identificar actividades de auditoría.

- Proporcionando a la administración y al cuerpo de PD la oportunidad de responder a los hallazgos de auditoría antes de su publicación.

- Haciendo patentes los aspectos positivos y negativos de las funciones de PD.

Siguiendo estas pautas, los auditores de PAD pueden hacer más fáciles sus labores y eliminar la relación antagónica que hace mucho tiempo ha estado asociada con las funciones de auditoría y de procesamiento de datos.

\section{EI PAPEL DE AUDITOR DE PAD EN EL DESARROLLO DE SISTEMAS}

El papel que juegan los auditores de PAD en el proceso de desarrollo de sistemas depende en gran parte de la naturaleza del ambiente así como del compromiso de la administración superior para asegurar que se genere un producto profesional con puntualidad y dentro del presupuesto. El precepto de que los auditores de PAD no deberían participar en la selección o el diseño del sistema que ellos intervendrán debe ser reconsiderado en vista de la tecnología de las computadoras de hoy.

Algunos auditores de PAD creen que su función está limitada a la revisión de aplicaciones del sistema después de que se ha completado el proceso de desarrollo; algunos hasta creen que su independencia y objetividad estarán comprometidas si participan activamente en el desarrollo del sistema. Este punto de vista parece ahora ceder su puesto a la creencia de que la participación temprana de la auditoría es la clave para asegurar que se incluyan los controles apropiados en el diseño de un nuevo sistema. Los proponentes de este punto de vista arguyen que como es muy oneroso modificar los sistemas de aplicación después de completados, los auditores de PAD tienen poca oportunidad de afectar la adecuación de los controles a menos que participen en el desarrollo de los sistemas. Estos auditores afirman que no se perderá objetividad si la responsabilidad operacional de los controles internos es puesta en donde reside, es decir, en los usuarios.

Los auditores de PAD deben aumentar sus responsabilidades tomando papeles activos en el proceso de desarrollo de los sistemas. Esta forma de mantenimiento preventivo de auditoría asegurará que el sistema propuesto satisfaga los objetivos de la empresa así como también las necesidades de los usuarios.

\section{Pruebas de programas y de sistemas}

Los programadores generalmente prueban un programa o un grupo de sub-programas a la vez. Luego los combinan con otros programas y con el tiempo ligan y prueban todos los programas que constituyen la corriente de trabajo.

Finalmente, los distintos trabajos se prueban en una serie lógica hasta que el sistema entero haya sido probado. Los auditores de PAD deberían estar envueltos en este proceso para verificar que estas pruebas son, en efecto, adecuadas y que las rutinas claves funcionan tal como fueron diseñadas. También deberían poder identificar los controles relacionados con elementos de datos y con funciones principales para así participar en la revisión previa a la implantación.

\section{Revisión previa a la implantación}

Esta revisión puede definirse como una fase independiente que precede a la implantación planeada del sistema y que sigue a la terminación de las pruebas de la lógica de programas. El papel del auditor de PAD en esta revisión es el de determinar si los requisitos del sistema han sido interpretados adecuadamente, transcritos 
a especificaciones de programación y ejecutados cuidadosamente para probar en todas las condiciones y situaciones posibles. También deben verificarse la exactitud y ejecución completa de todos los procedimientos rutinarios.

Si se dirige en forma apropiada, la revisión previa a la implantación puede servir de base para la revisión posterior a ella, determinando si los controles y procedimientos del sistema:

- Son compatibles con los objetivos del diseño.

- Requieren más investigación de auditoría.

- Indican riesgo significativo.

Aunque la extensión de la revisión previa a la implantación varía según la empresa, debe brindar siempre la perspectiva conveniente para planear y satisfacer la revisión posterior a la implantación.

\section{Revisión posterior a la implantación}

Esta revisión debe llevarse a cabo después de que se han eliminado todos los costos de desarrollo e implantación y una vez que todos los problemas han sido resueltos a satisfacción de los usuarios.

Según el Instituto de Auditores Internos (Institute of Internal Auditors), los objetivos de la auditoría de PAD en la revisión posterior a la implantación comprenden la determinación de:

- El costo real del desarrollo del sistema.

- Los beneficios que son y/o serán informados a consecuencia del nuevo sistema.

- El rendimiento económico estimado con base en los costos y beneficios identificados.

- Las razones de cualquier discrepancia entre los costos, los beneficios y la tasa de rendimiento económico reales con los estimados al inicio y durante el proyecto.

La comparación de los costos y de los beneficios reales con los presupuestos a menudo señalan con precisión deficiencias tales como malas técnicas de estimación, uso deficiente del equipo y del personal de PD, técnicas inapropiadas de programación y de pruebas, y la falla de los usuarios para aprovechar plenamente todas las ventajas del sistema.

\section{MÁS ALLA DE LA AUDITORÍA DE PAD}

Los auditores de PAD ven su función como una extensión más allá de las revisiones tradicionales anterior y posterior a la implantación.
Se dan cuenta de que deben separar los hechos de la ficción y demostrar un conocimiento de las prioridades de la administración para que sus hallazgos y recomendaciones reciban la atención apropiada. Además, los auditores deben tratar de motivar a la administración para que corrija errores y deficiencias al mismo tiempo que mantienen controles eficaces.

\section{Separar los hechos de la ficción}

Mediante el uso de una combinación de técnicas de auditoría probadas (entrevistas, pautas de estándares industriales, valores de ponderación y valores de medida), los auditores de PAD pueden separar eficazmente los hechos de la ficción. Sin embargo, quienes sean investigados deben percibir la metodología de la auditoría como apropiada, consistente y correcta, y a los auditores como idóneos y objetivos. La metodología de la auditoría, sin embargo, debe ser suficientemente flexible para acomodarse a los conceptos, al equipo y a las técnicas de procesamiento de datos nuevos o cambiantes.

Ya que la confianza en los hallazgos de auditoría es de suma importancia, los auditores de PAD deben demostrar su idoneidad para separar los hechos de la ficción:

- Participando en el proceso de desarrollo del sistema para garantizar que las características de auditoría y de control sean diseñadas en los nuevos sistemas.

- Dirigiendo revisiones periódicas de los sistemas operativos posteriores a su implantación para garantizar que cualquier modificación no perjudique la disponibilidad para efectos de auditoría.

- Adoptando nuevos instrumentos y técnicas de auditoría para que corran parejas con el uso creciente del procesamiento de datos y la introducción de nueva tecnología.

\section{Control del desempeño de los sistemas}

La naturaleza dinámica del procesamiento de datos demanda que los auditores PAD controlen regularmente el funcionamiento de los sistemas establecidos desde hace mucho tiempo y de los sistemas instalados recientemente. El control del desempeño de los sistemas puede variar desde una medida de tecleos por hora hasta una revisión detallada de la utilización del computador durante períodos de procesamiento en línea y en lotes. 
El auditor de PAD puede descontinuar el control de los sistemas operacionales una vez que se han corregido las condiciones deficientes y puede alejarse de los sistemas nuevos una vez que se han alcanzado exitosamente los puntos de prueba apropiados.

\section{La revisión semestral}

Hay varías escuelas de pensamiento en lo que concierne al intervalo que debe mediar entre la terminación de la auditoría de PAD y la conducción de las revisiones de seguimiento. Algunos auditores de PAD prefieren esperar a la auditoría del año próximo para la revisión de seguimiento, mientras que otros sostienen que seis meses de tiempo es suficiente para que el procesamiento de datos lleve a cabo sus recomendaciones. Hay argumentos válidos para ambos puntos de vista; sin embargo, un semestre debería proveer el tiempo suficiente para determinar el éxito de las acciones tomadas a consecuencia de los hallazgos iniciales de la auditoría de PAD.

Los auditores de PAD deberían usar esta revisión semestral como una oportunidad para:

- Garantizar que la documentación de todo el sistema esté completa y al día.

- Revisar el respaldo del sistema y los procedimientos de recuperación contra desastres.

- Reiterar los objetivos de la auditoría y poner de relieve su papel en el soporte de los esfuerzos futuros de diseño del sistema.

\section{CURSO DE ACCIÓN RECOMENDADO}

Comúnmente los auditores de PAD no penetran al ambiente de procesamiento de datos sino hasta después de que los sistemas son operacionales y la exposición al riesgo ya es clara. Participando desde el comienzo en el proceso de desarrollo de los sistemas y garantizando que ellos están diseñados, probados y documentados adecuadamente, los auditores pueden suministrar a la administración un análisis y una evaluación completa -con la esperanza de una evaluación efectiva cambiará la percepción del Auditor como un adversario del administrador.

\section{NOTAS}

1. Stanford Reaserch Institute, ahora SRI Internacional.

2. The Institute of Internal Auditors Inc. (1975). Hatching the EDP Audit. Funnctions. Orlando.

3. Lo ocurrido en recientes historias de horror de sociedades mercantiles puede hacer maravillas en la demostración de la necesidad de la función de auditoría y de la integridad del auditor.

\section{BIBLIOGRAFÍA}

COOPER LYBRAND E INSTITUTO DE AUDITORES INTERNOS DE ESPAÑA (1997). Nuevos conceptos del control interno. España.

CODERRE, DAVID (1998). Tools for de new role of audit. Canadá, ACL Services Ltd.

GÓMEZ AYALA, MARÍA (1998). Auditoría de calidad en la empresa moderna: un conducto hacia el camino cultural. México, Editorial Panorama.

PETER, NORTON (1999). Introducción a la computación. Mc Graw Hill Interamericana Editores S.A. México D.F..

PIATTINI, MARIO; PESO, EMILIO (1998). Auditoría informática: un enfoque práctico. México, Editorial Alfa Omega. 